\title{
Prescribing Practices by Nurse Practitioners and Primary Care Physicians: A Descriptive Analysis of Medicare Beneficiaries
}

\section{Authors: Ulrike Muench, Jennifer Perloff, Cindy Parks Thomas, and Peter I. Buerhaus}

NOTICE: this is the author's version of a work that was accepted for publication in Journal of Nursing Regulation. Changes resulting from the publishing process, such as peer review, editing, corrections, structural formatting, and other quality control mechanisms may not be reflected in this document. Changes may have been made to this work since it was submitted for publication. A definitive version was subsequently published in Journal of Nursing Regulation, Vol. 8, Iss. 1, (April 2017) DOI\#10.1016/S2155-8256(17)30071-6

Muench, Ulrike, Jennifer Perloff, Cindy Parks Thomas, and Peter I. Buerhaus. "Prescribing Practices by Nurse Practitioners and Primary Care Physicians: A Descriptive Analysis of Medicare Beneficiaries." Journal of nursing regulation 8, no. 1 (April 2017): 21-30.

Made available through Montana State University's $\underline{\text { ScholarWorks }}$ scholarworks. montana.edu 


\title{
Prescribing Practices by Nurse Practitioners and Primary Care Physicians: A Descriptive Analysis of Medicare Beneficiaries
}

\author{
Ulrike Muench, RN, PhD; Jennifer Perloff, PhD; Cindy Parks Thomas, PhD; and Peter I. Buerhaus, RN, PhD
}

\begin{abstract}
Introduction: Nurse practitioner (NP) prescribing continues to be a contentious policy issue, and studies systematically examining NP prescribing are lacking. The aim of this study was to conduct a descriptive analysis comparing the prescribing services of NPs with those of primary care physicians (PCPs) in providing care to Medicare beneficiaries. Methods: Part D drug claims of beneficiaries who saw an NP or a PCP in 2009 and 2010 were examined for differences in the types of medications prescribed, the volume of prescriptions, and the duration of prescriptions across all drug classes in Medicare Part D. Results: Data for 164,681 beneficiaries were analyzed. Results showed the same top 20 types of medications and the same share of generic medications for NP and PCP prescriptions. Differences in prescribing patterns were found for the number of prescriptions and for the duration of the prescriptions (days' supply per claim). NP beneficiaries received, on average, approximately one more 30-day prescription per year than PCP beneficiaries. The mean duration for an NP prescription claim was 3 days shorter than that for a PCP prescription claim, indicating that NP beneficiaries need refills sooner than PCP beneficiaries. This pattern existed in most drug classes and was more pronounced in behavioral drug classes, such as antidepressants, antipsychotics, psychotherapeutics, and opioids and in patients with more comorbidities. Differences in state scope of practice laws did not affect these prescribing patterns. Conclusions: Key differences were observed in the number and duration of prescriptions written by NPs and PCPs. Further research is needed to understand the impact of differences in NP and PCP patient populations on provider prescribing, such as the higher number of prescriptions issued by NPs for beneficiaries in moderate and high comorbidity groups and the implications of the duration of prescriptions for clinical outcomes, patient-provider rapport, costs, and potential gaps in medication coverage.
\end{abstract}

Keywords: Nurse practitioners, prescribing patterns, primary care physicians, research

0 hortages of physicians have been forecasted for primary care and specialty areas (IHS Inc, 2015). These shortages are particularly worrisome because nearly 80 million baby boomers will become eligible for Medicare over the next two decades, many of whom suffer from several comorbidities and degenerative diseases that will challenge the capacity of the Medicare program. Additionally, private, state, and federal efforts to increase access to care have resulted in approximately 20 million individuals gaining insurance coverage, and another 10 million or more are expected in the coming years. Thus, policymakers have become increasingly supportive of expanding the numbers, roles, and productive capacity of the primary care nurse practitioner (NP) workforce.

A growing body of research has established that NPs provide similar and, in some cases, superior care compared with primary care physicians (PCPs) (Lenz, Mundinger, Kane, Hopkins, \& Lin, 2004; Mundinger et al., 2000; Naylor et al., 2004; Newhouse et al., 2011; Venning, Durie, Roland, Roberts, \& Leese, 2000), and a recent national study of Medicare beneficiaries showed that the cost of primary care provided by NPs is substantially lower than care provided by PCPs (Perloff, DesRoches, \& Buerhaus, 2015). Yet, little is known about the medication prescribing practices of NPs (Lenz, Mundinger, Hopkins, Lin, \& Smolowitz, 2002; Shell, 2001). Prescription drug costs have increased sharply in recent years, including Medicare prescription drug costs (Sarpatwari, Avorn, \& Kesselheim, 2016), refocusing conversations on provider prescribing patterns and choices (DeJong et al., 2016). Concerns over the volume and costs of behavioral health medications and controlled substances have surfaced (Roehrig, 2016), highlighting the importance of assessing the prescribing practices of primary care providers at the forefront of managing behavioral health conditions (Hercelinskyj, Cruickshank, Brown, \& Phillips, 2014). Understanding how prescribing services differ between NPs and PCPs will help inform state and federal policymakers concerned 
with expanding the primary care workforce and curbing prescription drug costs (Sarpatwari, Avorn, \& Kesselheim, 2016).

This study made use of a representative sample of Medicare beneficiaries, allowing for a nationwide assessment of services provided by NPs and PCPs. The aims were to provide an overview of the types and volumes of medications prescribed by NPs and PCPs and to explore their prescribing patterns.

To examine medication use of Medicare beneficiaries, the authors analyzed data from Medicare Part D, the prescription drug coverage program for those covered under Medicare A or B. Drug coverage varies substantially among different Part D insurance plans, with differences in deductibles and coinsurance. Because little is known about NP prescribing services in Medicare data, the authors also examined whether three Medicare Part D coverage phases - initial benefit phase, coverage gap phase (also called the donut hole), and the catastrophic coverage phase-differ for beneficiaries receiving care from NPs versus PCPs.

\section{Methods}

This study was a retrospective descriptive analysis of prescription drug claims in a cohort of Medicare beneficiaries who received care by NPs and PCPs with national provider identification (NPI) numbers in 2009 and 2010. Institutional review board approval was obtained from research ethics boards at Vanderbilt University and the University of California San Francisco.

A prescription claim is a billing record registered in the Part D data system and indicates a new prescription or refill. The authors used the Medi-span proprietary therapeutic classification system (TCS) to quantify Part D claims and prescriptions for drug groups at different levels of aggregation, allowing identification of the top 20 drug classes, or up to 536 drug groupings. To examine prescribing practices, the authors focused on two key concepts in prescribing: the mean number of prescriptions and the duration of prescriptions. For example, for a medication to be taken continuously, one provider may give a patient a 90-day supply of a single prescription, and another may prescribe the same medication in three 30-day prescriptions. In analyzing the data, the authors observed the first as one claim and the latter as three claims, respectively.

An underlying assumption is that a systematic difference in the duration of a prescription may affect patient outcomes. For example, differences in prescription duration may have clinical implications because the patient needs to act to obtain refills or a new prescription to take medications continuously. This action may consist of a phone call or a message in an online portal, or it may be more involved, requiring a visit to the provider. At a minimum, a trip to the pharmacy to obtain the medication is required. Thus, a shorter duration could provide an opportunity for an important phone or in-person check-up, or it could lead to an unnecessary gap in medication coverage.

\section{Data}

The core analysis is based on beneficiaries' 2010 Medicare Part D data. The authors also used the beneficiary summary file to obtain beneficiary enrollment information in Part A, B, and D and dual Medicaid-Medicare eligibility status. In addition, the authors used Part B data to measure how the NP and PCP cohorts differed by clinical severity based on the Elixhauser comorbid conditions classification (Elixhauser, Steiner, Harris, \& Coffey, 1998). Because Medicare Part D claims do not include information on the drug class, the authors used data from Medi-span to categorize medications based on the National Drug Code.

\section{Sample}

The sampling strategy follows closely the work of Perloff and colleagues (2015). Beneficiaries were identified based on a random sample of NPs (specialty code "50") and PCPs (specialty code 08 = family medicine and $11=$ internal medicine). The sampling goal was to draw a cohort of approximately 1 million beneficiaries of NP and PCPs. The sample included 9,422 NP providers and 68,069 PCPs, representing approximately $80 \%$ of all Medicare NP providers and approximately $20 \%$ of all PCPs who offer services to Medicare beneficiaries. The authors purposefully oversampled beneficiaries who saw NPs to achieve adequate representation of NP services in the data because NPs see approximately half as many Medicare beneficiaries as PCPs (Perloff et al., 2015). The authors obtained all Part A, B, and D claims for the year 2010 from these beneficiaries, which resulted in a large number of claims belonging to both NPs and PCPs, as well as to other clinicians not in primary care, such as physician specialists.

Figure 1 illustrates the sample selection process. From the beneficiaries assigned to NPs or PCPs, (explained below) the authors selected those who had continuous enrollment in Part A, $\mathrm{B}$, and $\mathrm{D}$; did not switch prescription plans during the year; filled at least one prescription; and had no missing data in the demographic variables. For beneficiaries who died during 2010, the authors excluded their prescription claims from 6 months before their death because the goal was to capture the prescription profile of a typical primary care population. This decision was based on the rationale that major health changes likely take place before the end of life, possibly resulting in prescription drug changes. Finally, patients under 65 years of age were excluded from the analysis, as younger dual eligibles may have clinical profiles that are different than the rest of the Medicare population.

\section{Attribution}

To examine differences in services by NPs and PCPs, the authors assigned each beneficiary to a single provider type. The authors used the beneficiaries' total evaluation and management (E\&M) expenditures over a 12-month period to determine which provider was associated with the highest beneficiary expenditures and limited the E\&M expenditures to those identifiable as primary care: Berenson-Eggers Type of Service Codes for established office visit 
(M1B: 99211-99215), home visit (M4A: 99340-99345; 9934799350), or nursing home visit (M4B: 99404-99306; 99307-99310) (Perloff et al., 2015). Limiting attribution to claims from E\&M services from primary care service codes helps ensure that the clinicians in this study are providing substantial primary care services to Medicare beneficiaries.

Attribution of beneficiaries to NPs and PCPs was carried out in three steps. First, the authors calculated the proportion of primary care E\&M paid amounts for each beneficiary for each clinician in the 2009 data. Second, the beneficiary was assigned to the primary care clinician with the highest proportion of primary care E\&M costs. Third, to ensure a strong relationship between beneficiaries and primary care clinicians, the authors limited the sample to beneficiaries who received at least $30 \%$ of their services from one clinician. If two clinicians were associated with the same E\&M paid amounts, one clinician was selected at random.

\section{Variables}

To identify whether differences existed in the types of medications prescribed by NPs and PCPs, Medi-span's TCS was applied. This system provides a crosswalk of National Drug Codes to drug classes at different levels of granularity. Using the TCS, the authors identified the 20 most frequently prescribed medication classes in the data.

To measure the quantity of medications prescribed, the authors calculated the number of prescriptions filled in 2010 by standardizing prescription drug claims to a 30-day supply, as is standard in the literature (Medicare Payment Advisory Commission, 2015). "Days' supply" is a data field filled out by pharmacists, and 30-day supply was obtained by dividing by 30 the number of days for which a medication was prescribed. For example, a 45 -days supply on one prescription claim counted as 1.5 prescriptions.

\section{Prescribing Practices}

To discern the differences in provider prescribing patterns, the authors examined the relationships among number of prescriptions, number of claims, and days' supply using Medi-span's most granular TCS, categorizing medications into 536 drug groups.

Drug categories with fewer than 10 beneficiaries for NPs were not included to ensure adequate beneficiary representation in all drug categories. The resulting 307 drug groups were highly specific. For example, antidepressant drug group included alpha-2 receptor antagonists (tetracyclics), monoamine oxidase inhibitors, serotonin modulators, selective serotonin reuptake inhibitors, tricyclic agents, miscellaneous antidepressants, and antidepressant combination products. Comparing prescribing behaviors of clinicians in specific medication groups helps control for clinical differences among patients for which these medications are indicated.

The goals were to examine if differences in the number of prescriptions persisted in specific drug groups and to identify if NPs and PCPs write prescriptions for longer or shorter durations,
FIGURE 1

\section{Sample Selection Flow Diagram of Medicare Beneficiaries Receiving Care from Nurse Practitioners (NPs) and Primary Care Physicians (PCPs)}

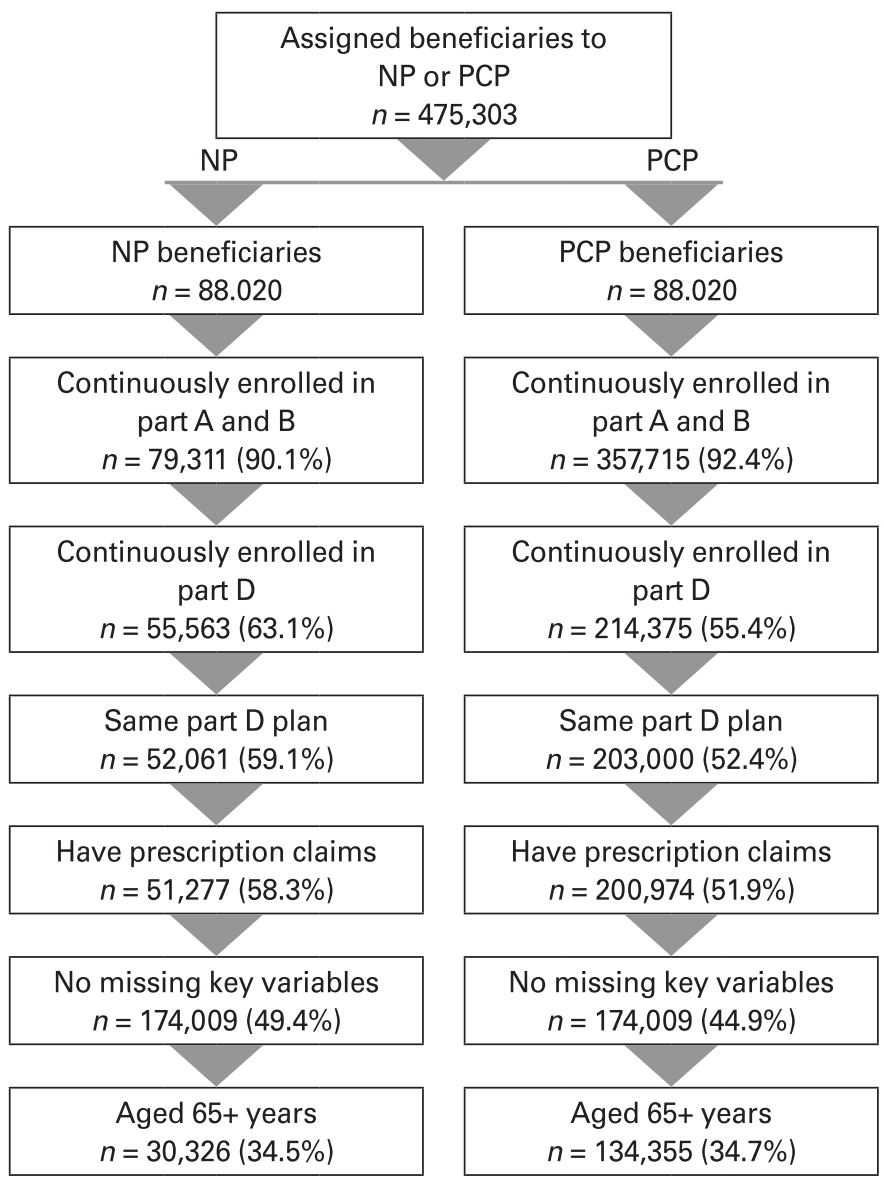

thus providing insight into whether patients seen by NPs and PCPs interact more or less often with the health care system for their prescription needs.

\section{Brand and Generic Medications}

The authors used the Medi-span brand and generic indicator variable to calculate the share of brand generic medications by NPs and PCPs.

\section{Comorbidity Adjustment of Clinical Severity}

Medicare administrative Part B claims include information on beneficiaries' pre-existing conditions in the form of diagnoses codes. The authors used this information and the Elixhauser comorbid conditions classification (Elixhauser et al., 1998), an algorithm that groups diagnoses codes into 30 comorbidities, to create three comorbidity groups of low, moderate, and high clinical severity: 0 to 3 comorbidities, 4 to 6 comorbidities, and more than 6 comorbidities. 
TABLE 1

\section{Sample Characteristics of Beneficiaries by Provider Type}

Variable

NP (\%) PCP (\%) p value

Demographics

\begin{tabular}{llll}
\hline White & 82 & 87 & $<.001$ \\
\hline Mean age (years) & 77.53 & 78.05 & $<.001$ \\
Female & 72 & 67 & $<.001$ \\
\hline Dual eligibility for Medicare/Medicaid & 46 & 31 & $<.001$ \\
\hline Rural & 5 & 3 & $<.001$ \\
CMS region & & & \\
$\quad$ Region 1 & 4 & 5 & $<.001$ \\
\hline Region 2 & 8 & 13 & $<.001$ \\
\hline Region 3 & 30 & 27 & $<.001$ \\
Region 4 & 16 & 20 & $<.001$ \\
\hline Region 5 & 13 & 8 & $<.001$ \\
\hline Region 6 & 9 & 7 & $<.001$ \\
Region 7 & 3 & 2 & $<.001$ \\
\hline Region 8 & 4 & 6 & $<.001$ \\
\hline Region 9 & 6 & 4 & $<.001$ \\
\hline
\end{tabular}

\section{Elixhauser Comorbidities}

Valve disorder

Pulmonary circulation disease

Peripheral vascular disease

Hypertension

Complex hypertension

Paralysis

Other neurologic disorders

Chronic pulmonary disease

Diabetes without chronic

complications

Diabetes with chronic complications

Hypothyroidism

Renal failure

Liver disease

Peptic ulcer disease and bleeding

Acquired immune deficiency

syndrome

Lymphoma

Metastatic cancer

Solid tumor without metastasis

Rheumatoid arthritis/collagen vas

Coagulopathy

Obesity

Weight loss

Fluid and electrolyte disorders

Chronic blood loss anemia

Alcohol abuse

Drug abuse

Psychoses

Depression

Elixhauser Comorbidity Groups

0-3 Comorbidities

4-6 Comorbidities

$>6$ Comorbidities

12

3

3
23

23

84

\section{5}

4

19

24

44

17

17
22
12

12

2

0

0

1

1

1

7

4

4

4

8

6

16

26

1

1

13

18

20

20

42

17

43

$<.001$

$38 \quad 39<.001$

Note. $\mathrm{CMS}=$ Centers for Medicaid and Medicare Services; NP = nurse practitioner; $\mathrm{PCP}=$ primary care physician.

\section{Statistical Analysis}

The authors conducted a descriptive analysis of beneficiaries and their prescription claims by provider type. When examining the number of prescriptions for the most common drug classes, the authors added the number of comorbidities of beneficiaries as another grouping variable to account for differences in illness severity in NP and PCP beneficiaries. This resulted in subanalyses by the three comorbidity groups. The authors tested for bivariate associations using $t$ tests and chi-square analyses for continuous and categorical data as appropriate to identify differences among providers on the study's prescribing outcomes of interest at a statistical significance level of $p<.05$. The number of prescriptions was skewed, and the authors used a Kruskal-Wallis nonparametric test for non-normally distributed data.

\section{Results}

\section{Sample Selection}

The analytic sample consisted of 164,681 beneficiaries; $18 \%$ ( $\mathrm{n}=30,326)$ were assigned to NPs, and $82 \%$ were assigned to PCPs $(n=134,355)$. According to the sample criteria, these beneficiaries were continuously enrolled in Medicare Part A, B, and D; did not switch prescription drug plans during 2010; had no missing demographic information; were aged 65 years or older; and had at least one prescription in 2010. Figure 1 depicts how each step in the sample selection process affected the sample size for NP and PCP beneficiaries.

A larger share of NP beneficiaries was covered under Part D (63\% NP beneficiaries vs. $55.4 \%$ PCP beneficiaries), indicating that NP beneficiaries use full Medicare benefits at a higher rate. Applying the minimum age criteria of 65 years resulted in a larger percentage of NP beneficiaries than PCP beneficiaries being dropped from the sample. This decrease likely results from the higher share of the dual Medicare-Medicaid-eligible population younger than 65 years seen by NPs.

\section{Beneficiary Characteristics}

Beneficiaries assigned to NPs were significantly more likely to be nonwhite, younger, female, dually eligible for Medicare and Medicaid, and living in rural areas $(p<.001)$ (See Table 1). They were also statistically significantly less likely to have each of the Elixhauser comorbid conditions except paralysis, neurological disorder, AIDS, weight loss, alcohol abuse, drug abuse, and psychoses. Examining the number of comorbidities showed that a greater share of NP beneficiaries was in the lowest comorbidity group, suggesting that beneficiaries assigned to NPs maybe somewhat healthier than PCP beneficiaries. Beneficiaries assigned to NPs were significantly more likely to reach the later Part D coverage phases that are associated with high prescription costs (coverage gap phase: NPs $41 \%$ vs. PCPs $36 \%$; catastrophic phase: NPs $14.6 \%$ vs. PCPs $10.1 \%)$. 
TABLE 2 Total Number of Prescriptions and Average Number of Prescriptions Per Beneficiary for 20
Most Common Drug Classes

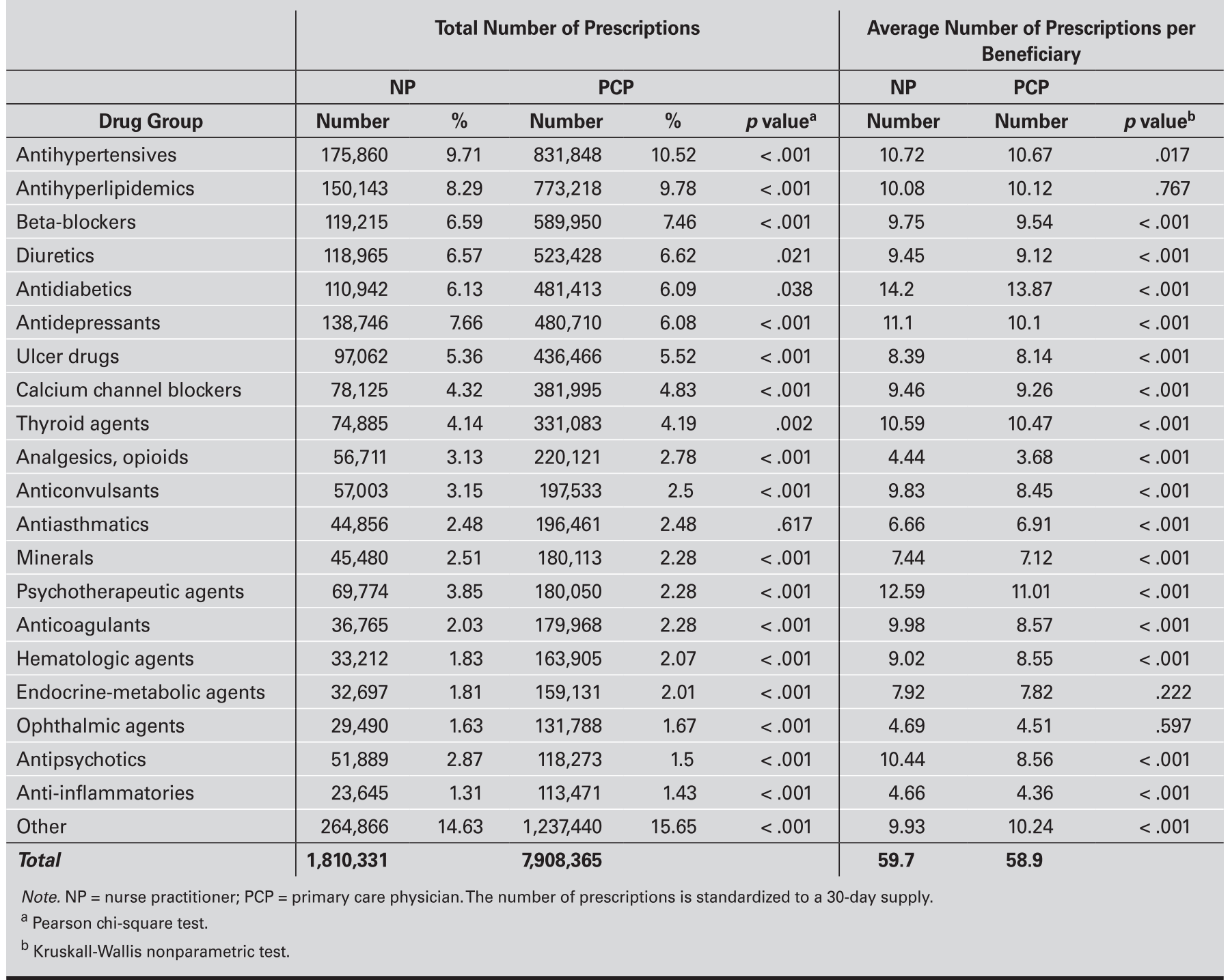

\section{Prescribing Characteristics}

Table 2 shows the total number of 30-day prescriptions filled for the 20 most common drug classes in 2010. The number of prescriptions in each drug class generated the same top 20 drug classes for both providers; however, the share of prescriptions for each class varied slightly across classes. The largest differences were for behavioral medications. NP beneficiaries filled a greater percentage of prescriptions for opioids (NPs 3.1\% vs. PCPs 2.8\%), psychotherapeutics (NPs $3.9 \%$ vs. PCPs 2.3\%), anticonvulsants (NPs 3.2\% vs. PCPs 2.5\%), antidepressants (NPs $7.7 \%$ vs. PCPs $6.1 \%$ ), and antipsychotics (NPs $2.9 \%$ vs. PCPs $1.5 \%$ ). In contrast, PCP beneficiaries filled a greater percentage of prescriptions for antihypertensives (NPs $9.7 \%$ vs. PCPs 10.5\%) and antihyperlipidemics (NPs $8.3 \%$ vs. PCPs 9.8\%). All drug groups tested as sig- nificantly different for NP and PCP beneficiaries at $p<.05$, except antiasthmatics.

\section{Provider Prescribing Patterns}

NP beneficiaries received on average 59.7 prescriptions in 2010 compared with PCP beneficiaries, who received 58.9 prescriptions, or approximately one additional 30-day prescription per year. This trend was consistent and statistically significant across most drug groups except for antihyperlipidemics, antiasthmatics, and endocrine and ophthalmic medications.

\section{Number of Prescriptions per Beneficiary and Beneficiary Comorbidities}

The authors conducted subanalyses by the number of comorbidities to address descriptively that beneficiaries attributed to PCPs 
TABLE 3

Average Number of Prescriptions Filled Per Beneficiary by Comorbidities: Top 20 Drug Classes

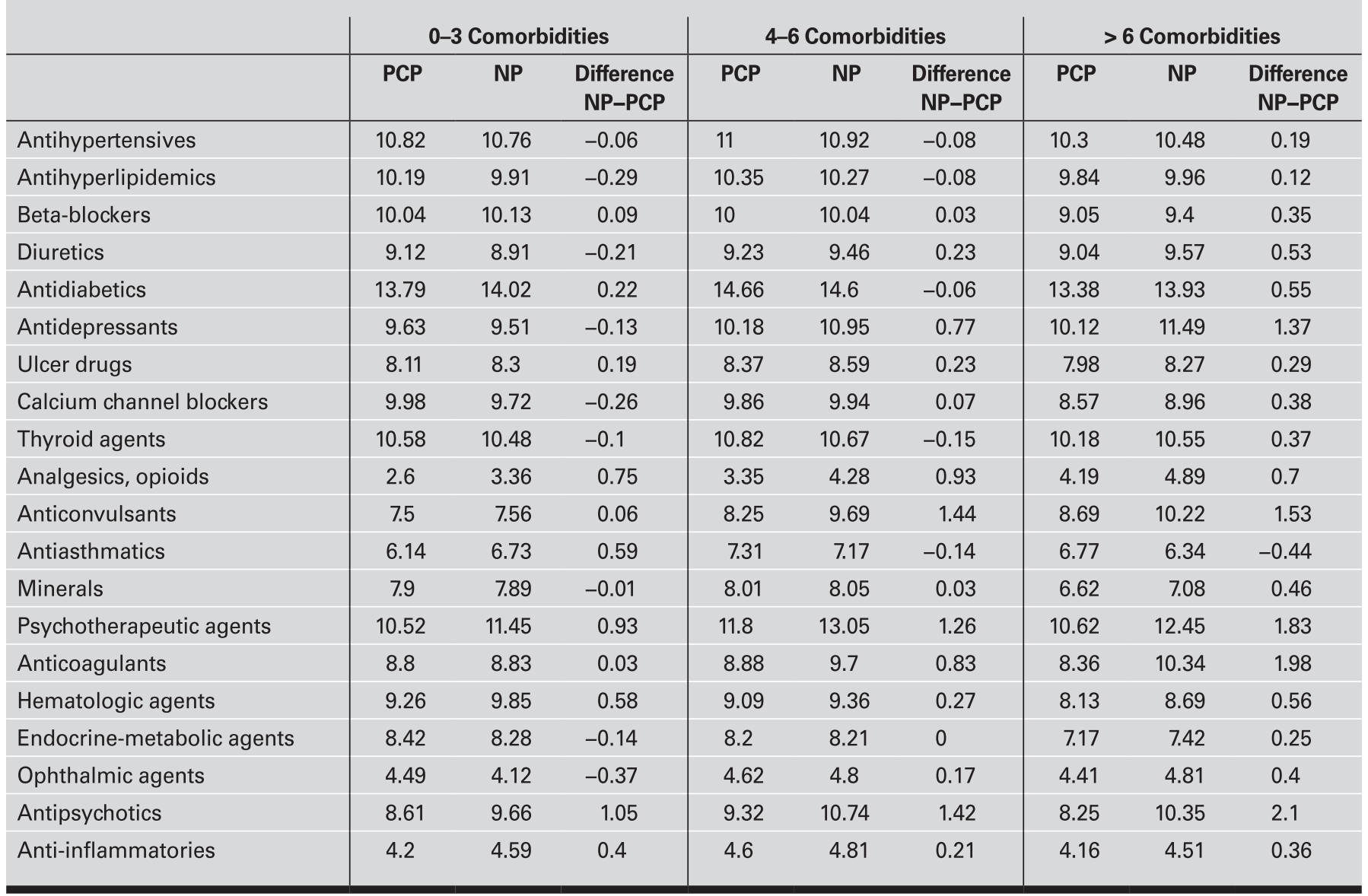

may be sicker and may thus require a different approach to drug management. Table 3 shows the number of prescriptions for the top 20 drug classes with subanalyses by the number of comorbidities. The difference between NP and PCP beneficiaries is indicated for each comorbidity group. The data suggest that, for those in the low comorbidity group, NP beneficiaries received slightly fewer prescriptions than PCP beneficiaries for approximately half of the drug classes, and, for those in the moderate and high comorbidity groups, NP beneficiaries received more prescriptions than PCP beneficiaries. In the high comorbidity group, NP beneficiaries filled more prescriptions in all but one drug class. Figure 2 plots the difference in the number of prescriptions for NP and PCP beneficiaries by comorbidity group. The difference in the number of prescriptions increased as the number of comorbidities increased, and this difference was more pronounced for behavioral drug classes.

\section{Number of Prescriptions, Number of Claims, and Days Covered per Prescription Claim}

Figure 3 illustrates differences between NP and PCP beneficiaries in four panels. Panel A shows the differences in the number of prescriptions for all 307 medication groups. Each line represents the positive differences between providers for each drug group. Panel B shows the positive difference of the number of days supplied between providers and illustrates that PCP beneficiaries receive a longer supply of a medication for $81 \%$ of all prescription drugs. Panel $\mathrm{C}$ plots the positive difference in number of claims for each drug group. For $80 \%$ of all medication groups, beneficiaries of NPs have more claims. Panel D illustrates the relationship between the number of prescriptions (Panel A) and number of claims (Panel C). The panel is ordered by number of prescriptions and shows the number of claims in light gray in the background. Panel D demonstrates that the number of prescriptions is unrelated to the number of claims because the pattern of the number of claims does not follow the contours of the number of prescriptions.

\section{Number of Prescriptions and State Scope of Practice Regulations}

The authors conducted follow-up analyses to examine if the general pattern of greater number of NP prescriptions was related to state scope of practice regulations. The authors categorized scope of practice legislations as independent prescribing and restrictive prescribing. The former included scopes of practice that allowed NPs to prescribe independently from physicians, including states that allowed NPs to prescribe schedule III drugs. The latter included 


\section{Difference in the Average Number of Prescriptions Between Nurse Practitioner and Primary Care Physician Beneficiaries by Number of Comorbidities: Top 20 Drug Classes}

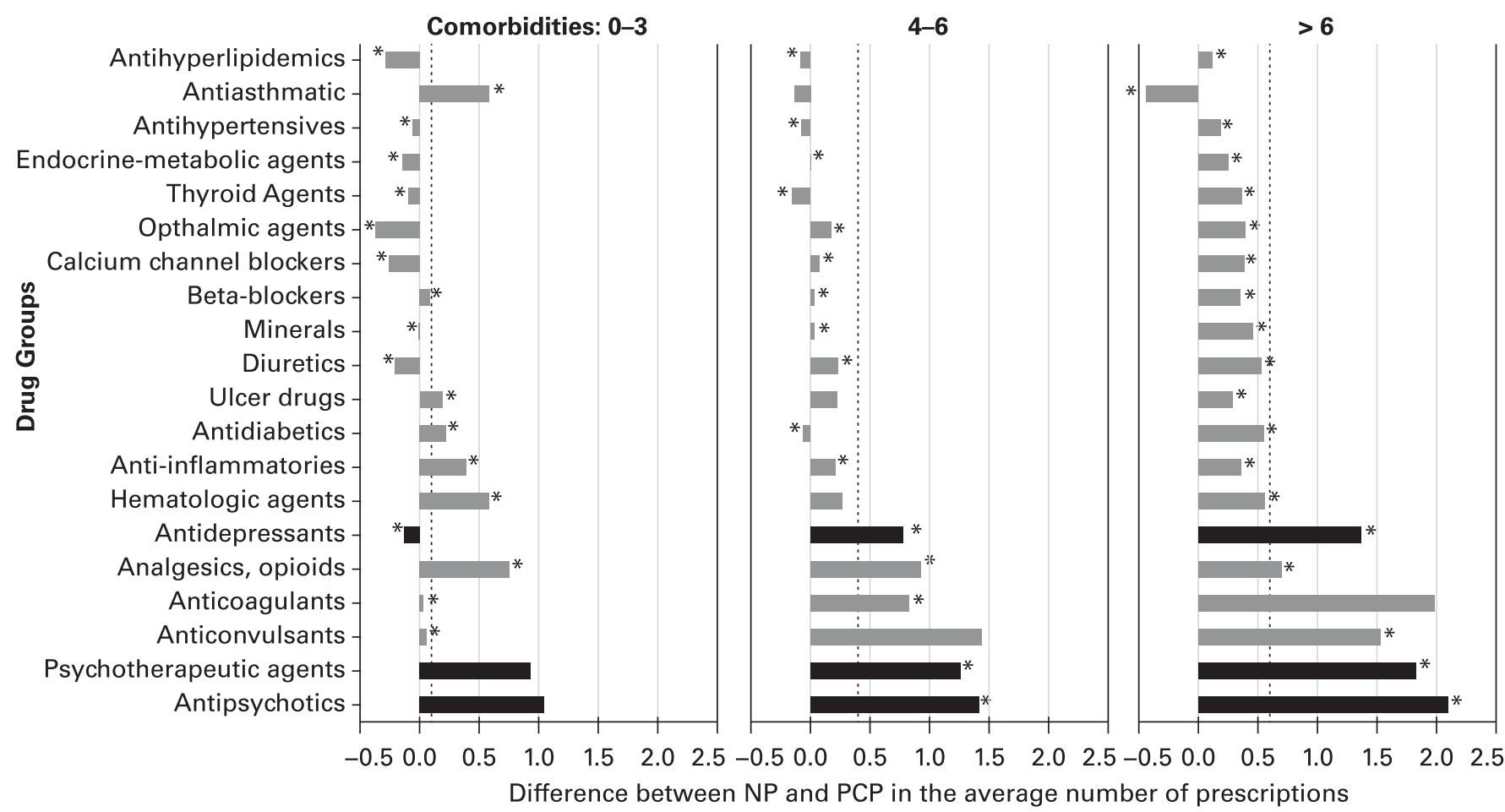

Behavioral medications

Other top 20 drug classes

Note. Prescriptions are standardized to a 30-day supply.

* $p<.05$

Dashed vertical lines indicate mean differences in number of prescriptions in each comorbidity group.

Drug groups are sorted by the size of the difference.

Drug groups in black indicate behavioral medication classes.

scopes of practice that require NPs to prescribe in collaboration with a physician or under a physician's supervision.

Figure 4 illustrates that NP beneficiaries receive more prescriptions from NPs under both types of scope of practice regulation. Because Figure 4 shows averages across states, the authors also calculated the difference in the number of prescriptions for NP and PCP beneficiaries for each state for the top 20 drug classes. The authors did not find that state scope of practice regulation significantly affected the trend of NP beneficiaries receiving more prescriptions (data not shown).

\section{Brand vs. Generic Medications}

The share of generic prescriptions was $72 \%$ for both NP and PCP beneficiaries. This percentage is approximately equivalent to 42 generic prescriptions per beneficiary out of 59 prescriptions for both provider types.

\section{Discussion}

Beneficiaries assigned to NPs were more likely to be in the later stages of the Part D drug benefit phase. Having more claims in the later benefit phases may indicate that a larger proportion of NP beneficiaries have conditions that require more expensive medications or that NP beneficiaries have clinical conditions that require more prescriptions. Together with the authors' findings on the higher volume of prescriptions for NP beneficiaries, these possible explanations require further study in a multivariate regression context, taking into account plan characteristics and other factors.

Analysis of the type of prescriptions indicated that the that the top 20 drug classes received by NP and PCP beneficiaries were similar. Further, both providers used generic prescriptions at essentially the same rate, which is not surprising because formularies, rather than individual providers, determine such choices.

The typical NP beneficiary received more prescriptions than the PCP beneficiary, and prescriptions and refills came with fewer days of medication coverage. Shorter days' supply on a claim suggests that refills must be requested more often if the medication 
FIGURE 3

\section{Relationship Among Number of Prescriptions, Number of Claims, and Average Days' Supply for NP and PCP Beneficiaries}
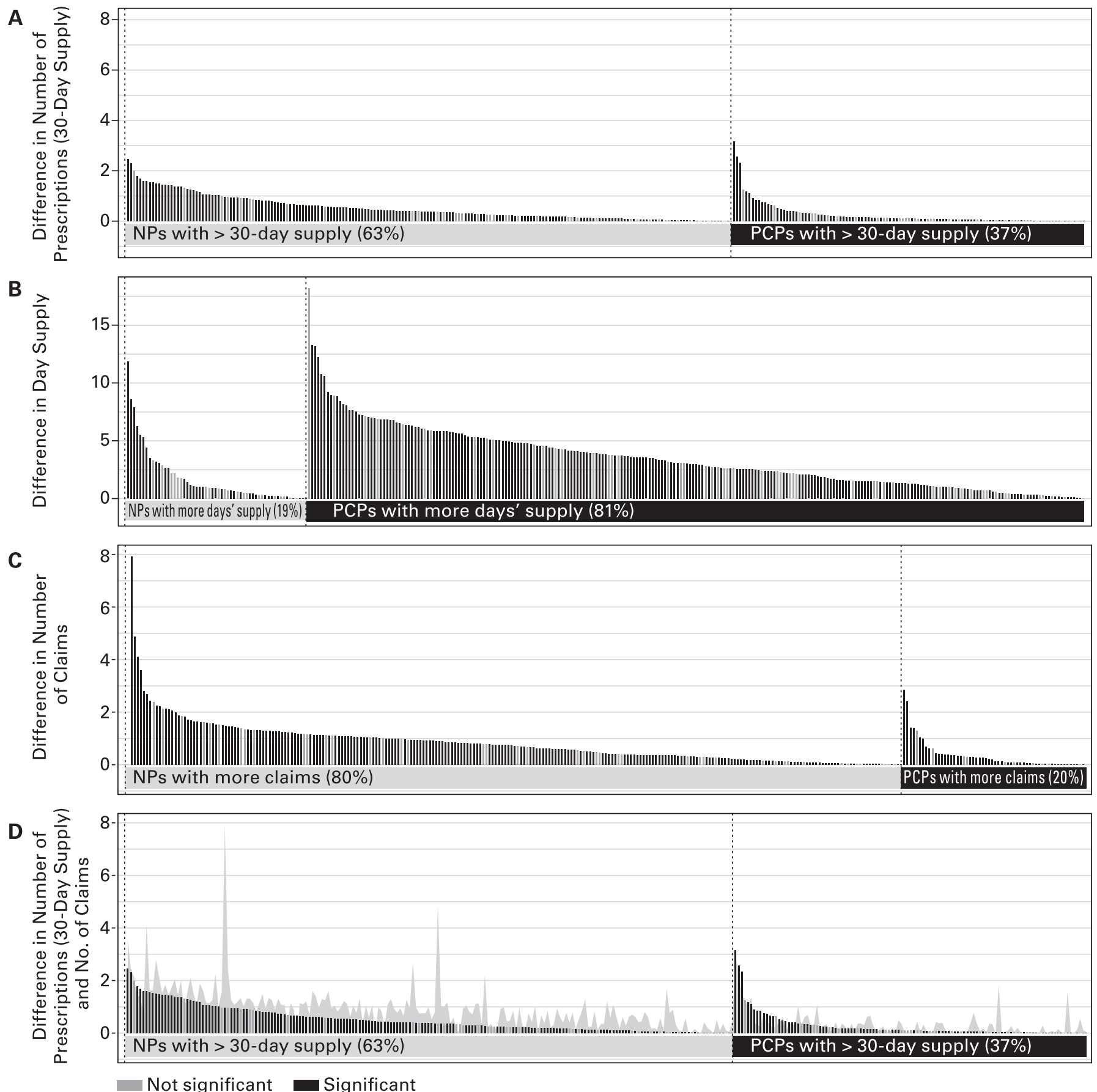

Note. Panels A through D show differences in study prescribing measures for nurse practitioner (NP) and primary care physician (PCP) beneficiaries for Medispan's smallest therapeutic classification system break down. Each bar represents one drug group. Bars colored in black indicate drug groups that were statistically different between NP and PCP beneficiaries at a significance level of $p<.05$. Only drug groups with $>10$ prescription claims for each provider type were included. Panel A shows differences in the number of prescriptions (standardized to 30-day supply) for all drug groups (sorted by the size of the difference in descending order). Panel B shows differences in the days' supply for each drug group (sorted by the size of the differences in descending order). Panel $\mathrm{C}$ shows the difference in the number of claims per beneficiary for each of the drug groups (sorted by the size of the difference in descending order). Panel D shows the difference in the number of prescriptions (standardized to 30-day supply), together with the difference in the number of claims (light gray) for each of the drug groups sorted by the number of prescriptions. 
is to be taken continuously, requiring communication with the primary care team, such as a telephone call or an online message. Contact with the provider at certain time intervals may be part of the pharmaceutical management plan. An office visit or a phone call provides an opportunity for the provider and the patient to communicate. For patients taking medications with high adverseeffect profiles, such communication may be necessary, and it offers an opportunity to strengthen patient-provider rapport. However, it could be an unnecessary burden to the patient, resulting in the patient running out of a medication sooner than necessary. A greater number of visits may also lead to increased administrative costs and time costs incurred by the patient.

\section{Limitations}

The authors followed an attribution approach that used claims from E\&M services belonging to primary care service codes to ensure that only NPs and PCPs working in primary care were part of the providers that were attributed to beneficiaries. Although the authors are confident that this approach facilitated the elimination of providers employed in specialty areas, some physician specialists and NPs working in specialist practices and clinics who billed for primary care services may be included in the provider pool.

The authors' analysis used administrative claims data, which includes NPs with their own NPI number. Thus, the analysis may not reflect all the Medicare services provided by NPs because those without their own NPI number who provide services for Medicare beneficiaries need to bill through another provider's NPI number, which could be an NP or a physician. However, even for the NPs with their own NPI numbers, services may be charged "incident to" a physician because Medicare reimbursement is higher for physicians than for NPs. Incident to billing is allowed when certain requirements are met, such as an initial encounter between a physician and a patient, and a physician's presence in the office when the NP sees patients (Shay, 2015). States that have restrictive NP scope of practice laws may have higher rates of incident to billing, but even in states where NPs can practice autonomously, incent to billing may occur because of the financial incentive of higher reimbursement. For these reasons, the study results do not reflect the services provided by NPs who do not have their own NPI number.

Finally, the results should be interpreted with the awareness that they are descriptive in nature and do not adjust for differences in patient characteristics between NP and PCP beneficiaries. The authors stratified the number of prescriptions by comorbidities (See Figure 2), which was the only partial adjustment. However, the authors analyzed medication groups at a fine level of aggregation using Medi-span's drug group classification to help ensure comparison of similar patients. The authors also examined whether the results were sensitive to state scope of practice laws and independent NP prescribing versus collaborative NP-physician prescribing and did not find that scope of practice regulations significantly affected the prescribing patterns of providers observed in these data. Although the authors explored this association only for the
FIGURE 4

\section{Difference in Number of Prescriptions Between Nurse Practitioners and Primary Care Physician Beneficiaries by State Scope of Practice Laws: Top 20 Drug Classes}

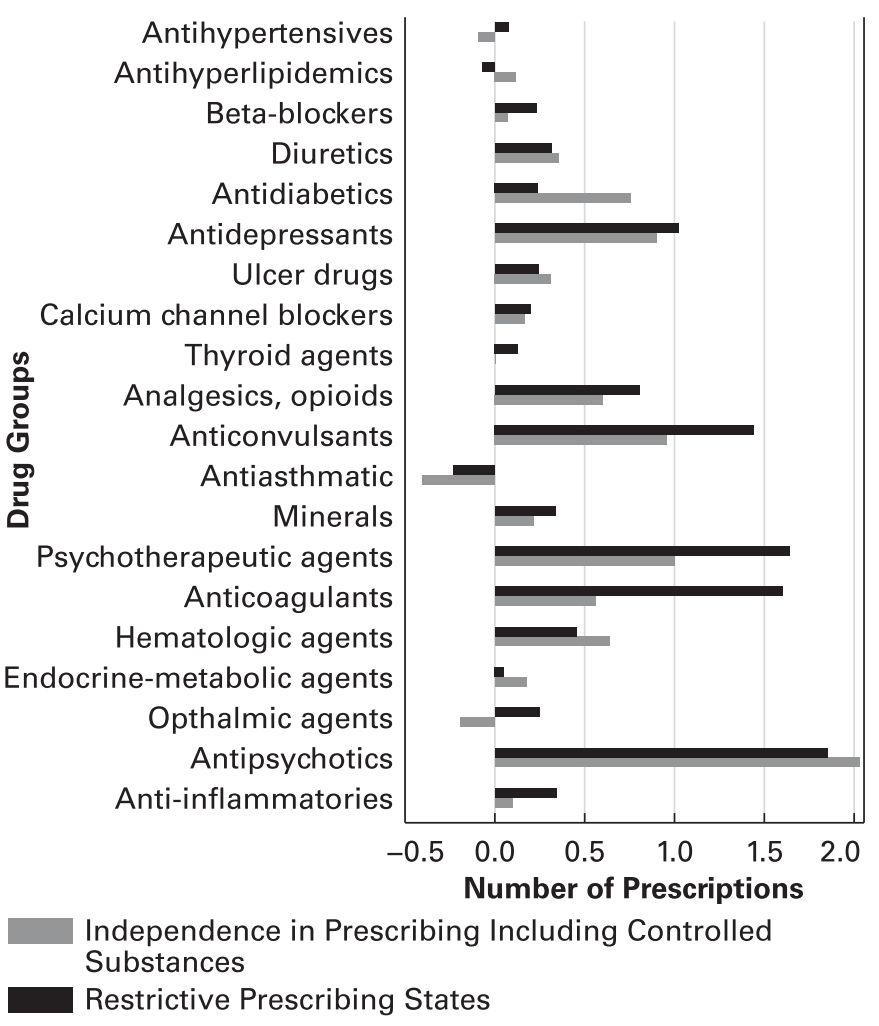

Note. The number of prescriptions is standardized to a 30-day supply. The bars to the right of zero indicate the positive difference in nurse practitioner prescriptions compared with primary care physician prescriptions.

20 most common drug classes, the prescribing patterns of NPs and PCPs were observed in the majority of states regardless of state scope of practice regulations. Thus, the prescribing practices of clinicians are probably not that influenced by scope of practice laws but might be related to individual clinician behavior and differences in practice styles between NPs and PCPs.

\section{Conclusion}

Key differences were observed in the number and duration of prescriptions written by NPs and PCPs. The findings warrant further study. Understanding how prescribing differences between NPs and PCPs affect patients clinically would emphasize the strengths of each type of provider and help guide the evaluation of NP scope of practice regulations. Such an understanding would also provide valuable information for workforce strategies aimed at increasing the capacity of the primary care workforce using NPs. 


\section{References}

DeJong, C., Aguilar, T., Tseng, C., Lin, G. A., Boscardin, W., \& Dudley, R. (2016). Pharmaceutical industry-sponsored meals and physician prescribing patterns for medicare beneficiaries. JAMA Internal Medicine. Retrieved from https://doi.org/10.1001/ jamainternmed.2016.2765

Elixhauser, A., Steiner, C., Harris, D. R., \& Coffey, R. M. (1998). Comorbidity measures for use with administrative data. Medical Care, 36(1), 8-27.

Hercelinskyj, G., Cruickshank, M., Brown, P., \& Phillips, B. (2014). Perceptions from the front line: Professional identity in mental health nursing. International Journal of Mental Health Nursing, 23(1), 24-32. Retrieved from https://doi.org/10.1111/inm.12001

IHS Inc. (2015). The Complexities of Physician Supply and Demand: Projections from 2013 to 2025. Prepared for the Association of American Medical Colleges. Washington, DC: Association of American Medical Colleges; 2015. Retrieved from https://www.aamc.org/download/426242/data/ihsreportdownload.pdf?cm_mmc=AAMC-_ -ScientificAffairs-_-PDF-_-ihsreport

Lenz, E. R., Mundinger, M., Hopkins, S. C., Lin, S. X., \& Smolowitz, J. L. (2002). Diabetes care processes and outcomes in patients treated by nurse practitioners or physicians. The Diabetes Educator, 28(4), 590 -598. Retrieved from https://doi.org/10.1177/ 014572170202800413

Lenz, E. R., Mundinger, M. O., Kane, R. L., Hopkins, S. C., \& Lin, S. X. (2004). Primary care outcomes in patients treated by nurse practitioners or physicians: Two-Year follow-up. Medical Care Research and Review, 61(3), 332 -351. Retrieved from https://doi.org/10.1177/ 1077558704266821

Medicare Payment Advisory Commission. (2015). Report to the Congress: Medicare and the Health Care Delivery System. Washington, DC.

Mundinger, M. O., Kane, R. L., Lenz, E. R., Totten, A. M., Tsai, W.-Y., Cleary, P. D., ... Shelanski, M. L. (2000). Primary care outcomes in patients treated by nurse practitioners or physicians. JAMA: The Journal of the American Medical Association, 283(1), 59-68. Retrieved from https://doi.org/10.1001/jama.283.1.59

Naylor, M. D., Brooten, D. A., Campbell, R. L., Maislin, G., McCauley, K. M., \& Schwartz, J. S. (2004). Transitional care of older adults hospitalized with heart failure: a randomized, controlled trial. Journal of the American Geriatrics Society, 52(5), 675-684. Retrieved from https://doi.org/10.1111/j.1532-5415.2004.52202.x

Newhouse, R. P., Stanik-Hutt, J., White, K. M., Johantgen, M., Bass, E. B., Zangaro, G., ... Weiner, J. P. (2011). Advanced practice nurse outcomes 1990-2008: A systematic review. Nursing Economics, 29(5), 1.

Perloff, J., DesRoches, C. M., \& Buerhaus, P. (2015). Comparing the cost of care provided to Medicare beneficiaries assigned to primary care nurse practitioners and physicians. Health Services Research, 51(4), 1407-1423. Retrieved from https://doi.org/10.1111/14756773.12425

Roehrig, C. (2016, June). Mental disorders top the list of the most costly conditions in the United States: $\$ 201$ billion. Health Affairs, Retrieved from https://doi.org/10.1377/hlthaff.2015.1659

Sarpatwari, A., Avorn, J., \& Kesselheim, A. S. (2016). State initiatives to control medication costs - Can Transparency legislation help? New England Journal of Medicine, 374(24), 2301-2304. Retrieved from https://doi.org/10.1056/NEJMp1605100

Shay, D. (2015). Using Medicare "Incendent-to" rules. Retrieved from http:// www.aafp.org/fpm/2015/0300/p15.pdf

Shell, R. C. (2001). Antidepressant prescribing practices of nurse practitioners. The Nurse Practitioner, 26(7, Part 1 of 2), 42.
Venning, P., Durie, A., Roland, M., Roberts, C., \& Leese, B. (2000). Randomised controlled trial comparing cost effectiveness of general practitioners and nurse practitioners in primary care. BMJ : British Medical Journal, 320(7241), 1048-1053.

Ulrike Muench, RN, PhD, is an Assistant Professor, Social and Behavioral Sciences, at the University of California San Francisco School of Nursing. Jennifer Perloff, PhD, is a Scientist at The Heller School, Brandeis University, Waltham, Massachusetts. Cindy Parks Thomas, PhD, is a Professor at The Heller School, Brandeis University. Peter I. Buerhaus, RN, PhD, is a Professor in the College of Nursing at Montana State University, Bozeman. 